\title{
Morphological defects of sperm and their association with motility, fertility, and hatchability in four Korean native chicken breeds
}

\author{
Shiferaw Garoma Feyisa', Young Hyun Park', Young Min Kim¹, Bo Ram Lee', Kyung Min Jung', \\ Seong Bok Choi ${ }^{2}$, Chang Yeon $\mathrm{Cho}^{2}$, and Jae Yong Han ${ }^{1, *}$
}

\begin{abstract}
* Corresponding Author: Jae Yong Han Tel: +82-2-880-4810, Fax: +82-2-874-4811, E-mail: jaehan@snu.ac.kr
\end{abstract}

1 Department of Agricultural Biotechnology and Research Institute of Agriculture and Life Sciences, College of Agriculture and Life Sciences, Seoul National University, Seoul 08826, Korea

${ }^{2}$ Animal Genetic Resources Research Center, National Institute of Animal Science, Rural Development Administration, Namwon 55717, Korea

ORCID

Jae Yong Han

https://orcid.org/0000-0003-3413-3277

Submitted Aug 22, 2017; Revised Oct 2, 2017; Accepted Nov 7, 2017
Objective: This study was conducted to compare morphological defects, viability, motility (MOT), fertility $(\mathrm{F})$, and hatchability $(\mathrm{H})$ in four Korean native chicken breeds (KNCBs), and to evaluate whether defective segments of spermatozoa are associated with MOT, F, and $\mathrm{H}$. Methods: Four KNCBs, including Korean Ogye (KO), Hwangbong (HB), Hyunin Black $(\mathrm{HH})$, and Hoengseong Yakdak (HY) were used. White Leghorn (WL) was used as a control. Nine cocks from each breed were randomly assigned into three groups. Semen was collected by abdominal massage method. Eosin-nigrosin staining method was used to identify livedead spermatozoa. Different segments and specific morphological defects of spermatozoa were identified using 4, 6-diamidino-2-phenylidole and MitoTracker Red CMXRos. F and $\mathrm{H}$ rates were evaluated following artificial insemination (AI).

Results: KO had the highest MOT rate compared to HY. Viable normal sperm rates of KO and $\mathrm{HH}$ were high and comparable with WL. HY spermatozoa had the highest viable abnormal sperm (VAS) or morphological defect rate followed by HB. Likewise, HB spermatozoa had the highest dead sperm (dead) rate compared to KO, HY, and WL. Bent, coiled, detached, broken, and knotted were common identified specific morphological defects for all breeds. Most morphological defects were at the head and tail in all breeds. VAS showed strong negative correlation with MOT $(\mathrm{r}=-0.697)$ and $\mathrm{F}(\mathrm{r}=-0.609)$. Similarly, defective tail was negatively correlated with MOT $(\mathrm{r}=-0.587), \mathrm{F}(\mathrm{r}=-0.797)$, and $\mathrm{H}(\mathrm{r}=-0.448)$. The $\mathrm{F}$ and $\mathrm{H}$ rates of $\mathrm{KO}$ and WL were comparable.

Conclusion: These data indicate that most identified specific morphological defects are at the head and tail. VAS and defective tail were associated with poor motility, F, and $\mathrm{H}$. KNCBs showed more morphological defects than WL. Finally, these results will facilitate successful $\mathrm{AI}$ and semen cryopreservation.

Keywords: Fertility; Hatchability; Native Chicken; Motility; Morphological Defect

\section{INTRODUCTION}

Assisted reproductive technologies such as artificial insemination (AI) and semen cryopreservation play an important role in preserving and transfusing valuable genes to the future generation and thus help to conserve and propagate endangered species which fail to breed in captivity [1]. The AI is one of the most effective and widely used techniques for the genetic improvement of farm animals [2]. It is employed on breeder farms to maintain the maximum use of males, as well as ensure disease prevention, high fertility rates and for economic reasons. AI is also carried out when natural mating is difficult in genetically improved animals due to body size [3]. However, the success of both AI and semen cryopreservation is directly dependent on semen quality. The importance of semen evaluation in poultry breeding for selecting breeding males or for routinely monitoring their reproductive performance is well 
recognized [4].

Recently, the demand for Korean native chicken breeds (KNCBs) increased suddenly as they suit the Korean palate and as the National institute of Animal Science (Korea) realized the necessity of conserving native breeds [5]. Thus, semen quality checking is required for successful semen conservation and AI. A scientific determination of the fertilizing ability of the semen can be made by motility, sperm concentration, live or dead counts, and morphological examinations $[2,6,7]$. Fertility and hatchability are important traits for the broiler breeder industry that can be affected by semen quality and are most sensitive to environmental and genetic influences [8]. The effect of age, chicken lighting schedule, season, body weight, and diet as well as semen collector on semen quality was highlighted recently [9]. Moreover, semen quality and quantity were reported to be affected by the breed and strain of chicken $[10,11]$.

Korean Ogye (KO), Hwangbong (HB), Hyunin Black (HH), and Hoengseong Yakdak (HY) are among a few KNCBs documented in the World watch list for domestic animal diversity of the Food and Agriculture Organization (FAO) [12]. However, there is no information on semen quality such as sperm motility, viability, and morphology in these breeds. Furthermore, information on sperm morphological defects and types and their subsequent association with motility, fertility, and hatchability is lacking in these breeds. Therefore, in this study, we investigated semen quality in four KNCBs using eosinnigrosin, 4', 6-diamidino-2-phenylidole (DAPI) and Mito Tracker Red CMXRos for estimation of dead, viable abnormal sperm (VAS) or morphological defect and viable normal sperm (VNS) in order to determine their association with motility, fertility and hatchability.

\section{MATERIALS AND METHODS}

\section{Experimental animal management}

Four KNCBs, such as KO, HB, HH, and HY breeds were used for this study. White Leghorn (WL) cocks were used as a control group. Forty-five cocks, 9 from each breed, and $60 \mathrm{WL}$ hens were randomly selected for semen collection and fertility and hatchability testing, respectively. The age of the experimental animals range from 48 to 60 weeks. Cocks of each breed were randomly divided into three groups $(n=3)$ and each group placed in a separate cage. Four WL hens were randomly allotted per group and placed in a separate cage. $\mathrm{KNCB}$ and WL chickens were maintained in a standard management program at the University Animal Research Farm, Seoul National University (SNU), Korea. Procedures involving animal management and reproduction, adhered to the standard operating protocols of our laboratory. Protocols for the care and experimental use of animals were approved by the Institute of Laboratory Animal Resources, SNU (SNU-150827-1).

\section{Semen collection}

Semen was collected from cocks of each different genetic group twice per week by using the abdominal massage method [13]. A single investigator did collection and examination of semen during the whole study period to avoid investigator bias.

\section{Sperm motility}

To determine the percentage of sperm motility (MOT), $2 \mu \mathrm{L}$ of undiluted semen was added to $100 \mu \mathrm{L}$ phosphate-buffered saline and mixed well. A drop of this mixture was placed on a pre-warmed microscope slide $\left(37^{\circ} \mathrm{C}\right)$, covered with glass slide, and observed using phase-contrast microscope. For each group, three experiments were independently made in order to obtain a single point (mean) and at least 300 sperms were counted per slide as described by Khan and Ijaz [14].

\section{Sperm viability and morphology}

Sperm viability refers to the percentage of live sperm in the semen sample, whereas morphology refers to the shape of the sperm cells. Head, midpiece, and tail of semen samples were observed for morphological variations. Sperm viability was microscopically evaluated by assessing at least 300 sperms per group for each breed under microscope.

\section{Eosin-nigrosin staining method}

One-step eosin-nigrosin staining technique [15] was used to evaluate the viability of spermatozoa. Briefly, $5 \mu \mathrm{L}$ of semen of each group was mixed with $20 \mu \mathrm{L}$ of eosin/nigrosin solution in a $0.6 \mathrm{~mL}$ graduated microcentrifuge tube (Simport, Beloeil, QC, Canada) [16]. The prepared semen samples were smeared on microscope slides and fixed by air-drying at room temperature for $10 \mathrm{~min}$ before observation. Viable sperm remained unstained and dead cells were totally or partially pink to red/ brown. Viable sperms were further classified as morphologically normal or abnormal, depending on the head, midpiece, and tail morphology [15].

\section{MitoTracker Red CMXRos staining method}

Nuclear and mitochondrial fluorescent dyes were used to identify different segments of the spermatozoa [17], and to show the morphological defects in these regions. Briefly, $1 \mathrm{~mL}$ of diluted semen (1:100) per replicate was incubated with 0.25 $\mu \mathrm{L}$ of MitoTracker Red CMXRos (Invitrogen, Carlsbad, CA, USA) at $37^{\circ} \mathrm{C}$ for $30 \mathrm{~min}$. Subsequently, the mixtures was centrifuged at $500 \mathrm{~g}$ for $1 \mathrm{~min}$. After the supernatants were discarded, samples were fixed with $10 \%$ formalin for $30 \mathrm{~min}$ at room temperature. Then, $10 \mu \mathrm{L}$ of samples (per group) were placed on microscope slides, air dried, and mounted in Prolong Gold antifade reagent with DAPI (Invitrogen, USA). Samples were observed under fluorescence microscope (Nikon, Eclipse Ti, Tokyo, Japan). Nuclear fluorescent dye stained the head region (blue), whereas MitoTracker Red CMXRos stained 
the midpiece and tail regions (red).

\section{Artificial insemination}

Each experimental hen received $100 \mu \mathrm{L}$ of its respective undiluted fresh semen twice per week for three to four weeks to evaluate the fertility and hatchability.

\section{Fertility and hatchability evaluation}

Eggs were collected and labeled separately by group for each breed and stored at $14^{\circ} \mathrm{C}$ to $16^{\circ} \mathrm{C}$ for 6 to 8 days after which they were incubated for fertility and hatchability evaluation. Fertility was calculated as the proportion of fertile eggs among incubated eggs, fertile eggs was determined by conventional candling method at 7 days after incubation, while hatchability was calculated as the proportion of hatched chicks among total incubated eggs.

\section{Statistical analysis}

Data were analyzed by using statistical program (IBM SPSS, version 23; SPSS Inc., Chicago, IL, USA). One-way analysis of variance was employed to determine an overall difference in dead, VAS, VNS, fertility, and hatchability between breeds. When main effects were determined to be statistically significant, the effect of breeds was compared using Duncan post hoc multiple comparisons. A p-value of less than 0.05 was considered significant. Pearson's correlation estimates were performed to determine the associations of defective segments with motility, fertility, and hatchability.

\section{RESULTS}

\section{Comparison of sperm motility and viability from KNCBs and WL}

Four KNCBs and WL chicken were used for this study and representative image of cocks from each breed is depicted in Figure 1. Mean percentage motility of sperm collected from $\mathrm{WL}, \mathrm{KO}, \mathrm{HB}, \mathrm{HH}$, and $\mathrm{HY}$ was $88.7 \% \pm 2.6 \%, 87.3 \% \pm 1.4 \%$, $85.0 \% \pm 3.6 \%, 83.3 \% \pm 2.0 \%$, and $78.3 \% \pm 0.9 \%$, respectively (Figure 2A). The MOT of HY was significantly low $(\mathrm{p}<0.05)$ compared to WL and $\mathrm{KO}$. However, there was no significant difference ( $p>0.05$ ) in MOT among HY, HB, and HH. Mean dead, VAS, and VNS of KNCBs and WL are given in Figure 2B. There were statistically significant differences among breeds in percentage of dead sperm. HB had higher $(\mathrm{p}<0.05)$ percentage of dead spermatozoa followed by HY. However, the percentage of dead spermatozoa in $\mathrm{KO}, \mathrm{HY}, \mathrm{HH}$, and WL did not show significant variation. The percentage of VAS in WL, $\mathrm{KO}, \mathrm{HB}, \mathrm{HH}$, and $\mathrm{HY}$ was $8.5 \pm 1.7,8.8 \pm 0.7,11.7 \pm 1.7,9.7 \pm 1.2$,
A

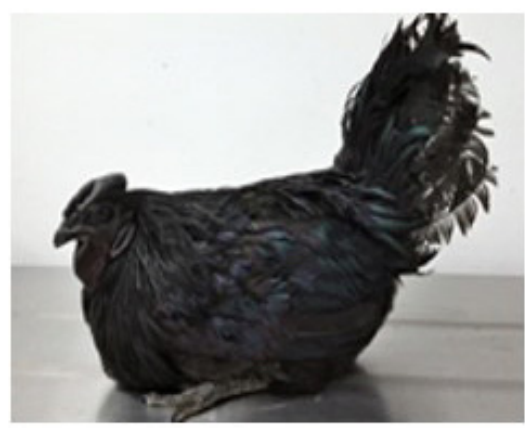

$\mathrm{D}$

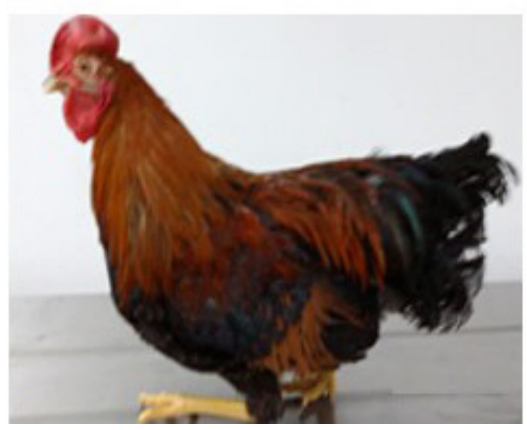

B

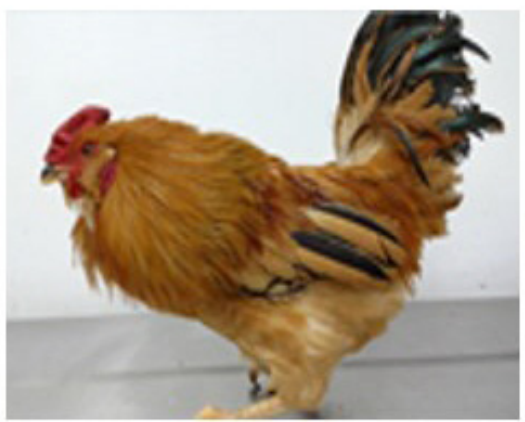

E

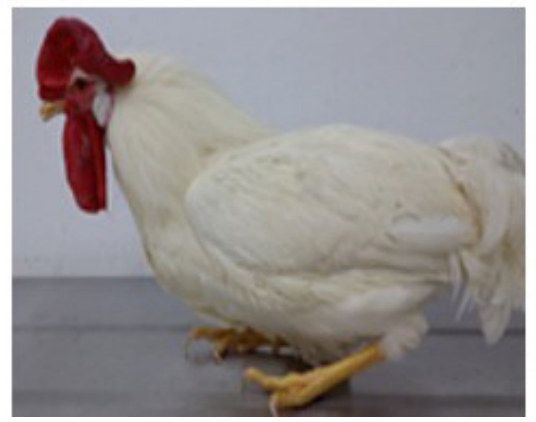

C

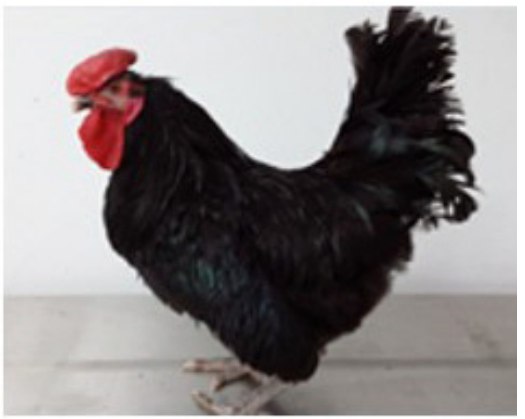

Figure 1. Representative images of experimental cocks used in this study. (A) Korean Ogye (KO), deep black feather and grey/black comb; (B) Hwangbong (HB), yellowish brown feather and bright red comb; $(\mathrm{C})$ Hyunin Black (HH), black feather and red comb; (D) Hoengseong Yakdak (HY), reddish brown feather and red comb; and (E) White Leghorn (WL), white feather and red comb. 
A. Motility

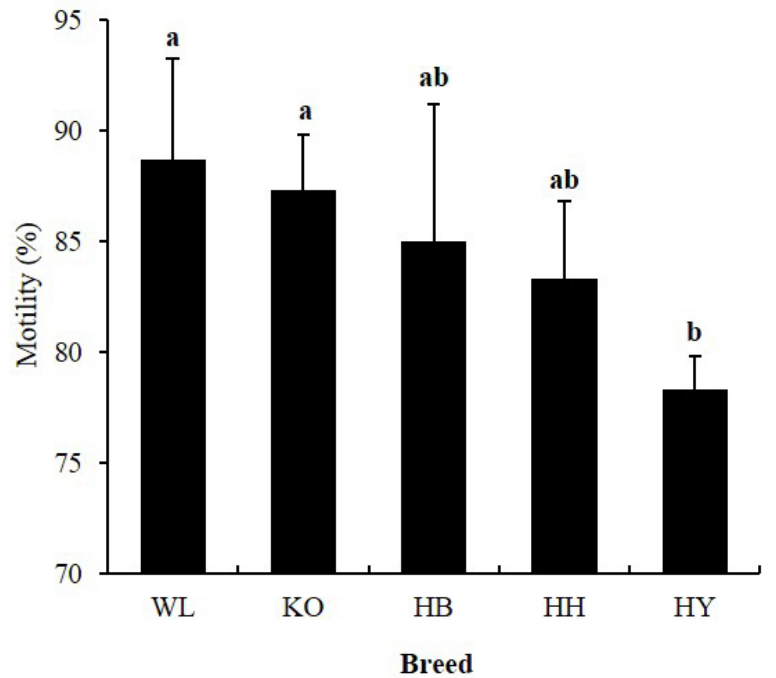

B. Live-dead and morphology

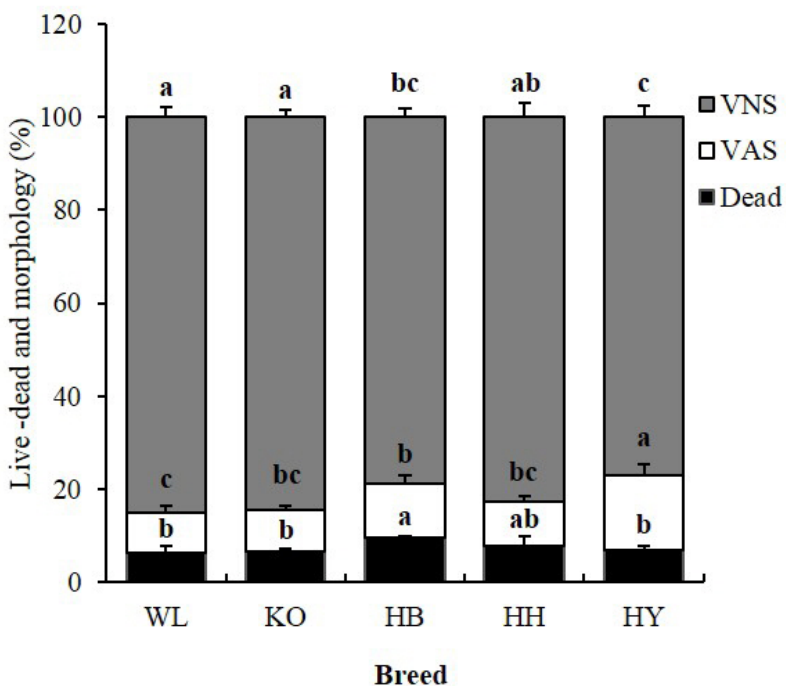

Figure 2. Effect of breed on sperm motility, viability and morphology in four Korean native and White Leghorn chicken breeds. (A) Sperm motility, (B) Sperm viability and morphology. Means with different letters are significantly different from each other $(\mathrm{p}<0.05)$, and error bars represent standard deviation of the mean. VNS; viable normal sperm, VAS; viable abnormal sperm, Dead; dead sperm.

and 16.0 \pm 2.4 , while the percentage of VNS was $85.1 \pm 1.2,84.5$ $\pm 0.9,78.8 \pm 1.1,82.5 \pm 1.8$, and $77.1 \pm 1.5$ for WL, KO, HB, HH, and HY, respectively. HY had higher percentage of $(p<0.05)$ VAS compared to all breeds, and lower percentage of VNS compared to $\mathrm{KO}, \mathrm{HH}$, and WL. Collectively, the data presented here indicates that $\mathrm{KO}$ and $\mathrm{HY}$ had the highest and the lowest motile spermatozoa among KNCBs, respectively. $\mathrm{KO}, \mathrm{HH}$, and WL spermatozoa had low percentages of dead/ VAS and high percentage of VNS. However, HB spermatozoa had high percentage of dead sperm, and HY spermatozoa had the highest VAS and the lowest VNS.

\section{Morphological examination of fresh spermatozoa from all breeds}

Morphological evaluation of fresh spermatozoa from four Korean native and WL chicken breeds were conducted using co-staining with DAPI and MitoTracker Red CMXRos. Representative images of morphological defects in the spermatozoa of four KNCBs and WL are shown in Figure 3. In this study, spermatozoa with bent, coiled, detached, broken, and knotted abnormality were observed in all breeds. The proportions of spermatozoa with total head, midpiece, and tail morphological defects in four KNCBs and WL are summarized in Figure
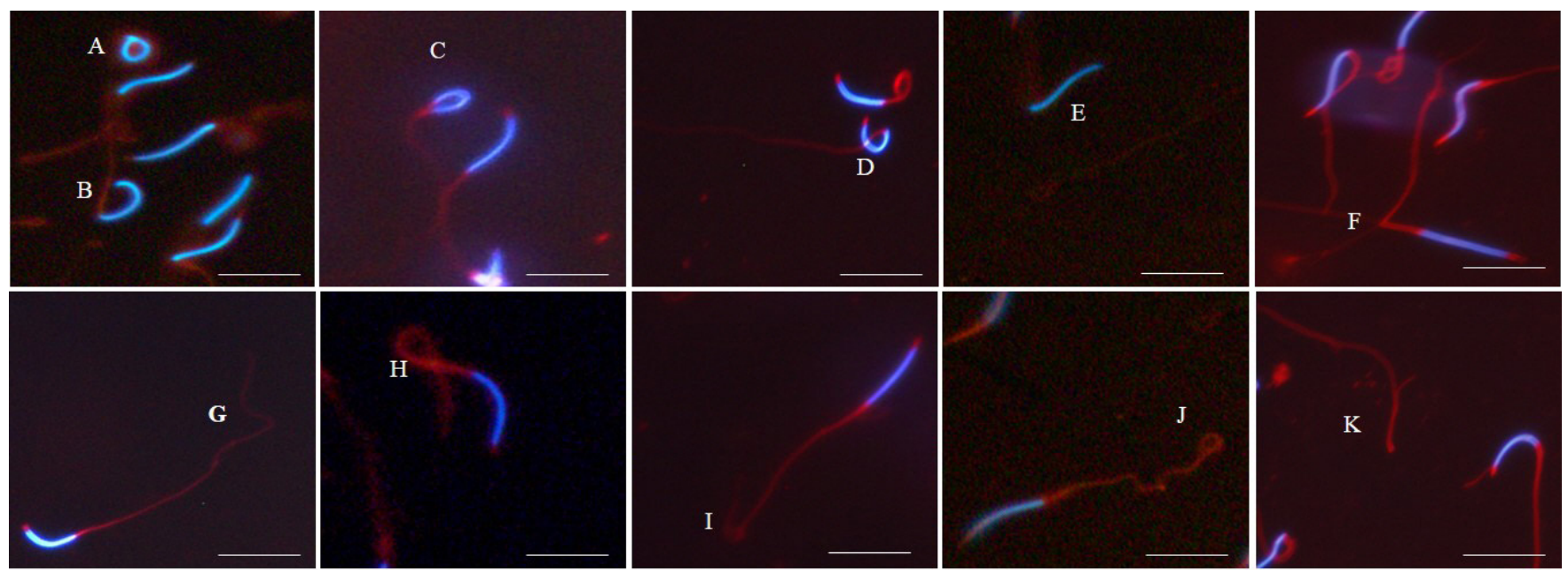

Figure 3. Representative images of morphological defects of fresh spermatozoa in four Korean native and White Leghorn (WL) chicken breeds. (A) coiled head, (B) bent head, (C) broken head, (D) Knotted head, (E) detached head, (F) broken midpiece, (G) bent tail, (H) knotted tail, (I) broken tail, (J) coiled tail, (K) detached tail. Samples were co-stained with 4', 6-diamidino-2-phenylidole (DAPI) and MitoTracker Red CMXRos. Nuclear fluorescent dye stained the head region (blue), whereas MitoTracker Red CMXRos stained the midpiece and tail regions (red). Scale bars: $20 \mu \mathrm{m}(\mathrm{A}, \mathrm{B}$, and $\mathrm{E})$ and $50 \mu \mathrm{m}(\mathrm{C}, \mathrm{D}, \mathrm{F}, \mathrm{G}, \mathrm{H}, \mathrm{I}, \mathrm{J}$, and $\mathrm{K})$. 


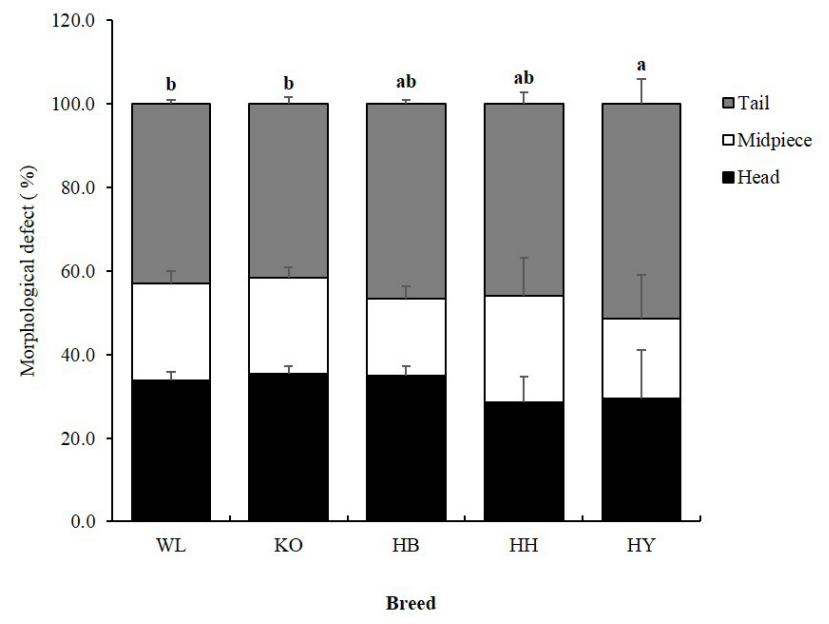

Figure 4. Percentage of total head, midpiece, and tail defective of spermatozoa in four Korean native and White Leghorn chicken breeds. Means with different letters are significantly different from each other $(p<0.05)$, and error bars represent standard deviation of the mean.

4. In the results, there was a statistically significant difference in the proportion of spermatozoa with defective segments among breeds. Most sperm abnormalities were found to occur at the tail followed by the head. Percentage of spermatozoa with tail abnormality in HY was significantly high $(\mathrm{p}<0.05)$ compared to WL and KO.

Head abnormalities were found to be the second most common defect in all breeds (Figure 4). There were statistically significant differences $(\mathrm{p}<0.05)$ in the percentage of spermatozoa with bent, coiled, detached, broken, and knotted head among breeds (Figure 5). The percentage of spermatozoa with bent and coiled head was higher in HY as compared to other breeds. Likewise, the percentage of spermatozoa with detached head in $\mathrm{HY}$ was significantly higher compared to $\mathrm{WL}$ and $\mathrm{KO}$, while the percentage of spermatozoa with broken head in $\mathrm{KO}$ was significantly higher compared to $\mathrm{HY}$ and $\mathrm{HH}$. Whereas, the percentage of spermatozoa with broken head in $\mathrm{HB}$ and $\mathrm{HH}$ was high compared to HY. Moreover, the percentage of spermatozoa with knotted head in HY was very low compared to $\mathrm{KO}, \mathrm{HB}, \mathrm{HH}$, and WL. However, $\mathrm{KO}, \mathrm{HB}, \mathrm{HH}$, and WL did not show significant variation for this defect.

Furthermore, we evaluated the proportion of spermatozoa with defective midpiece in all breeds. There were statistically significant differences $(\mathrm{p}<0.05)$ in the proportions of spermatozoa with bent, broken, and knotted defective midpiece among breeds (Figure 6). The percentage of spermatozoa with knotted midpiece was high in $\mathrm{KO}$ compared to $\mathrm{HB}$ and $\mathrm{HH}$. In addition, the percentage of spermatozoa with bent midpiece in $\mathrm{HH}$ was high compared to other breeds, whereas, the percentage of spermatozoa with knotted midpiece was very low in $\mathrm{HH}$ compared to KO, HY, and WL. The proportion of spermatozoa with bent and knotted midpiece was high and low, respectively, in KNCBs compared to WL.

There were statistically significant differences $(\mathrm{p}<0.05)$ in the proportions of spermatozoa with bent, coiled, detached,

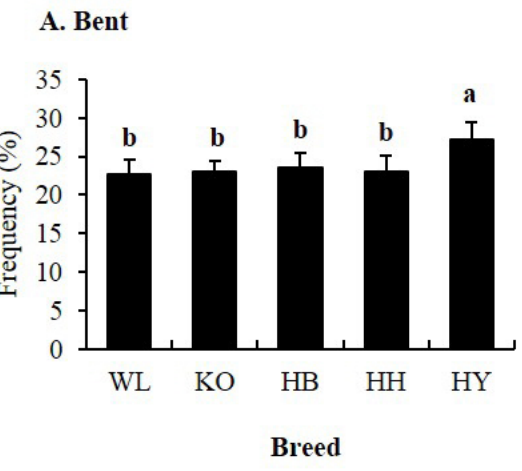

\section{Broken}

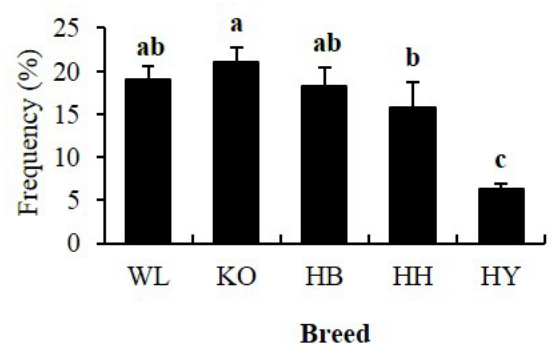

B. Coiled

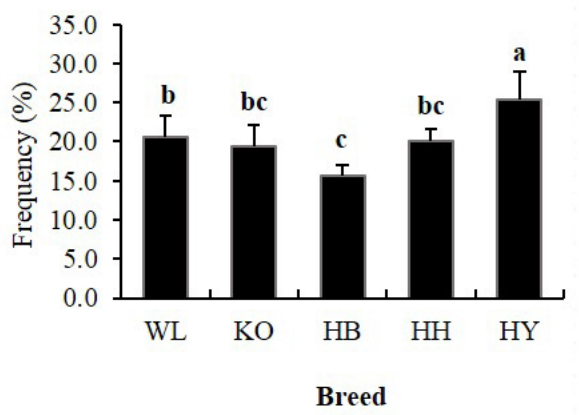

E. Knotted

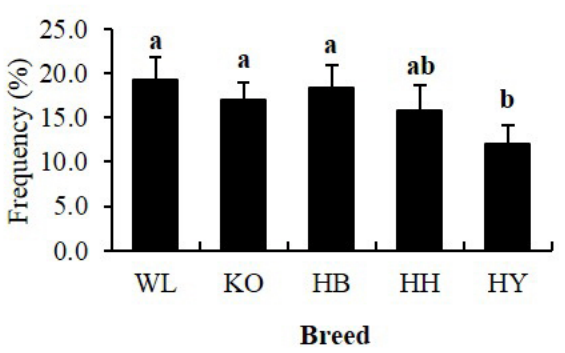

C. Detached

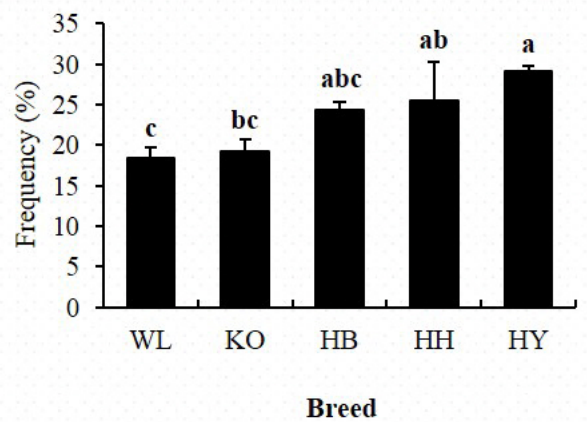

Breed

Figure 5. Percentage of specific head abnormalities of fresh spermatozoa in four Korean native and White Leghorn chicken breeds. (A) Bent, (B) Coiled, (C) Detached, (D) Broken, and $(E)$ Knotted. ${ }^{a, b, c}$ Means with different letters are significantly different from each other $(p<0.05)$, and error bars represent standard deviation of the mean. 

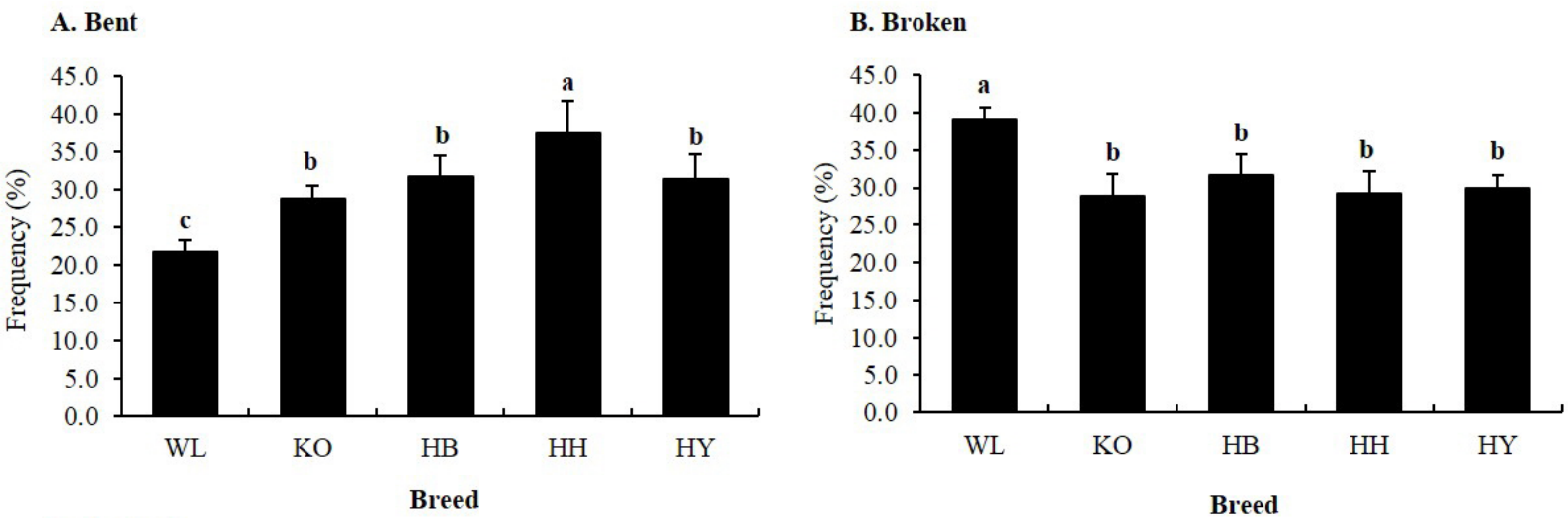

\section{Knotted}

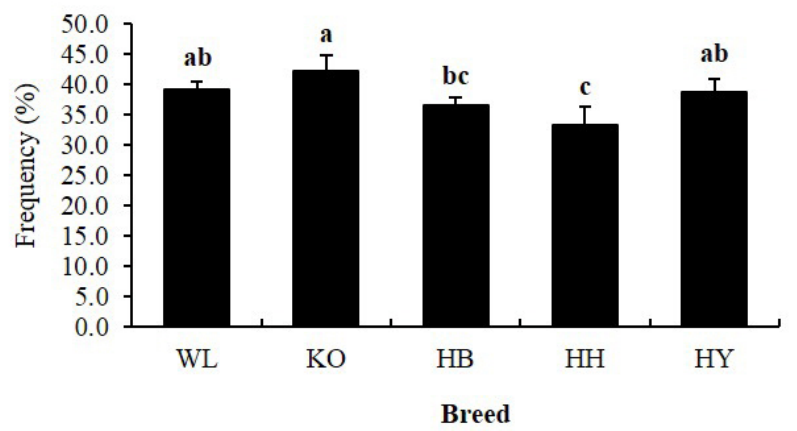

Figure 6. Percentage of specific midpiece abnormalities of fresh spermatozoa in four Korean native and White Leghorn chicken breeds. (A) Bent, (B) Broken, and (C) Knotted. ${ }^{a, b, c}$ Means with different letters are significantly different from each other $(p<0.05)$, and error bars represent standard deviation of the mean.

broken, and knotted tail among breeds (Figure 7). The percentage of spermatozoa with bent tail in $\mathrm{HH}$ and $\mathrm{HY}$ was significantly higher compared to HB and WL. However, $\mathrm{HH}$ had lower proportion of coiled tail defective spermatozoa com-
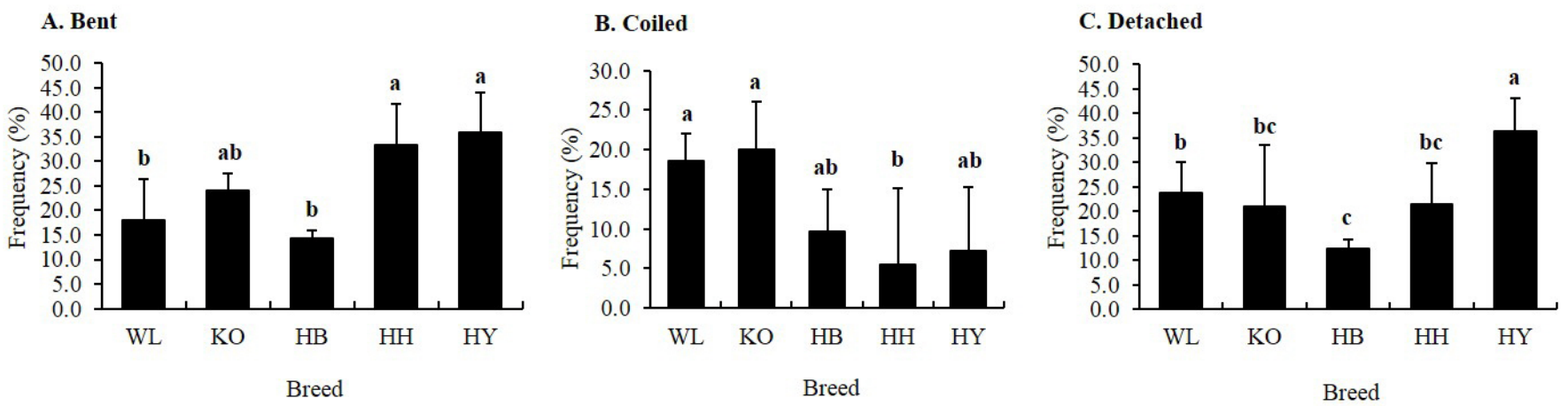

\section{Broken}

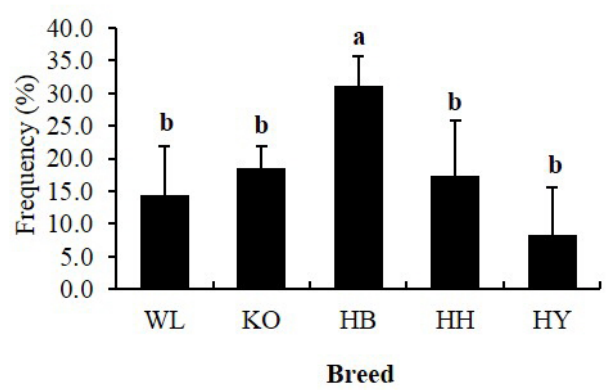

\section{E. Knotted}

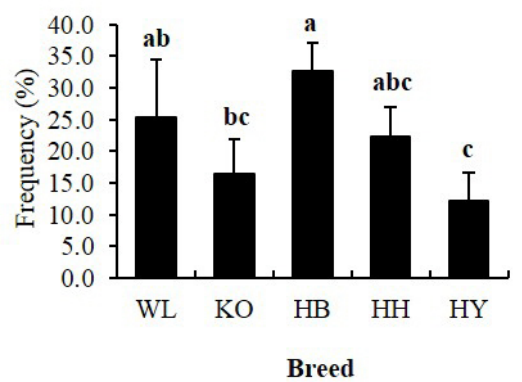

Figure 7. Percentage of specific tail abnormalities of fresh spermatozoa in four Korean native and White Leghorn chicken breeds. (A) Bent, (B) Coiled, (C) Detached, (D) Broken, and (E) Knotted. ${ }^{a, b, c}$ Means with different letters are significantly different from each other $(p<0.05)$, and error bars represent standard deviation of the mean. 
pared to KO and WL. The percentage of spermatozoa in HY with detached tail was high compared to all breeds. HB had the lowest percentage of spermatozoa with detached tail compared to $\mathrm{HY}$ and $\mathrm{WL}$. In contrast, percentage of spermatozoa with broken tail in $\mathrm{HB}$ was high compared to all breeds. Likewise, the percentage of spermatozoa with knotted tail in $\mathrm{HB}$ was high compared to $\mathrm{KO}$ and $\mathrm{HY}$.

\section{Fertility and hatchability testing}

The fertility rates of $\mathrm{WL}, \mathrm{KO}, \mathrm{HB}, \mathrm{HH}$, and $\mathrm{HY}$ were $96.4 \% \pm$ $0.6 \%, 94.9 \% \pm 1.4 \%, 91.6 \% \pm 1.8 \%, 90.4 \% \pm 0.5 \%$, and $87.8 \% \pm$ $1.0 \%$, respectively. Likewise, hatchability rates were $91.5 \% \pm$ $1.9 \%, 92.9 \% \pm 1.9 \%, 82.1 \% \pm 1.9 \%, 84.7 \% \pm 0.5 \%$, and $85.6 \% \pm 0.9 \%$ for WL, KO, HB, HH, and HY, respectively (Table 1). KO exhibited significantly higher fertility rate compared to $\mathrm{HH}$ and $\mathrm{HY}$ and significantly higher hatchability rate compared to other KNCBs. However, KO and WL did not show significant difference for both traits. Collectively, this data indicate that $\mathrm{KO}$ had best fertility and hatchability rates that are comparable with WL and superior to other native chicken breeds.

\section{Correlation of defective segments of spermatozoa, motility, fertility, and hatchability}

Summary of Pearson's correlation coefficient of defective head, defective midpiece, defective tail, motility, fertility, and hatchability of fresh spermatozoa in four KNCBs are given in Table 2 . The correlation of defective tail with MOT $(\mathrm{r}=-0.587)$, fertility $(r=-0.597)$, and hatchability $(r=-0.448)$ were strong and negative. Defective head and midpiece showed to have very weak positive correlation with MOT, fertility, and hatchability.

Correlation of dead, viable abnormal sperm, viable normal sperm, motility, fertility, and hatchability Pearson's correlation coefficient of dead, VAS, VNS, MOT, fertility, and hatchability of fresh spermatozoa in four KNCBs are given in Table 3. Dead sperm showed weak and negative correlation with MOT $(r=-0.206)$ and fertility $(r=-0.162)$

Table 1. Mean percentage of fertility and hatchability of fresh semen in four Korean native and White Leghorn chicken breeds following artificial insemination

\begin{tabular}{lccc}
\hline Breed & Eggs set (No.) & Fertility $^{1)}(\%)$ & Hatchability $^{2)}(\%)$ \\
\hline WL & 90 & $96.4 \pm 0.6^{\mathrm{a}}$ & $91.5 \pm 1.9^{\mathrm{a}}$ \\
KO & 100 & $94.9 \pm 1.4^{\mathrm{ab}}$ & $92.9 \pm 1.9^{\mathrm{a}}$ \\
HB & 88 & $91.6 \pm 1.8^{\mathrm{bc}}$ & $82.1 \pm 1.9^{\mathrm{b}}$ \\
HH & 72 & $90.4 \pm 0.5^{\mathrm{c}}$ & $84.7 \pm 0.5^{\mathrm{b}}$ \\
HY & 60 & $87.8 \pm 1.0^{c}$ & $85.6 \pm 0.9^{\mathrm{b}}$ \\
\hline
\end{tabular}

Values are mean \pm standard error of triplicate analyses.

KO, Korean Oge; HB, Hwangbong; HH, Hyunin Black; HY, Hoengseong Yakdak; WL, White leghorn.

1) Fertility, the percentage of total eggs set that is fertile.

2) Hatchability, the percentage of total eggs set that hatch.

$a, b, c$ Means within a column with different superscripts indicate significant difference $(p<0.05)$.
Table 2. Pearson's correlation coefficient of defective head, defective midpiece, defective tail, motility, fertility, and hatchability of fresh spermatozoa in four Korean native chicken breeds

\begin{tabular}{lcccccc}
\hline Variables & HDD & MPD & TLD & MOT & F & H \\
\hline HDD & 1 & - & - & - & - & - \\
MPD & $-0.742^{*}$ & 1 & - & - & - & - \\
TLD & -0.308 & -0.410 & 1 & - & - & - \\
MOT & 0.152 & 0.267 & $-0.587^{*}$ & 1 & - & - \\
F & 0.251 & 0.180 & $-0.597^{*}$ & $0.525^{*}$ & 1 & - \\
H & 0.120 & 0.200 & -0.448 & 0.301 & $0.750^{*}$ & 1 \\
\hline
\end{tabular}

HDD, defective head; MPD, defective midpiece; TLD, defective tail; MOT, motility; $F_{\text {, }}$ fertility; $H$, hatchability.

Tail abnormalities showed strong negative correlation with motility, fertility, and hatchability.

${ }^{*} p<0.05$.

whereas moderate and negative correlation with hatchability $(\mathrm{r}=-0.511)$. Similarly, VAS was negatively correlated with MOT $(r=-0.697)$, fertility $(r=-0.609)$, and hatchability $(r=$ -0.396). On the other hand, the correlation of VNS with MOT, fertility, and hatchability were positive and strong. The data presented here shows that motility, fertility, and hatchability are affected by defective tail and VAS.

\section{DISCUSSION}

Sperm quality has an important role for successful AI and cryopreservation $[18,19]$. In the present study, we evaluated and compared fresh sperm motility, viability, morphological defects, fertility, and hatchability in four Korean native and WL chicken breeds. In addition, we identified specific morphological defects on different segments of spermatozoa and evaluated the association of defective segments with motility, fertility, and hatchability.

In this study, variations in MOT, VAS, VNS, morphological

Table 3. Pearson's correlation coefficient of dead, viable abnormal sperm, viable normal sperm, motility, fertility, and hatchability of fresh spermatozoa in four Korean native chicken breeds

\begin{tabular}{lcccccc}
\hline Variables & DEAD & VAS $^{1)}$ & VNS $^{2)}$ & MOT & F & H \\
\hline DEAD & 1 & - & - & - & - & - \\
VAS & 0.099 & 1 & - & - & - & - \\
VNS & -0.338 & $-0.892^{* *}$ & 1 & - & - & - \\
MOT & -0.206 & $-0.697^{* *}$ & $0.700^{* *}$ & 1 & - & - \\
F & -0.162 & $-0.609^{*}$ & $0.647^{* *}$ & $0.525^{*}$ & 1 & - \\
H & -0.511 & -0.396 & $0.593^{*}$ & 0.301 & $0.750^{*}$ & 1 \\
\hline
\end{tabular}

Dead, dead sperm; VAS, viable abnormal sperm; VNS, viable normal sperm; MOT, motility; $F$, fertility; $H$, hatchability.

1) VAS: Any deviation from the normal structure (morphological defects, such as bent, coiled, detached, broken, and knotted were considered as an abnormal in this study).

${ }^{2)}$ VNS: Live sperm with normal structure.

VAS showed negative correlation with MOT, F, and H. VNS exhibited positive correlation with these traits.

${ }^{*} p<0.05 ;{ }^{* *} p<0.01$. 
defect, fertility, and hatchability rates were observed between breeds. The assessment of sperm motility is one of the most often used parameters for semen evaluation [20]. Sperm motility observed in the Korean breeds was within the range of the earlier reports for different chicken breeds [10,21,22]. HY had lower motility rate compared to WL and KO. However, the sperm motility rates obtained for all breeds in this study are sufficient to fertilize the egg as revealed in Table 1. Sperm viability is one of the most important semen quality parameters that are used to determine the sperm quality factor to indicate the fertilizing ability of the semen. Values reported for live normal sperm of KNCBs (77.1\% to $84.5 \%$ ) and WL $(85.1 \%)$ in this study are similar to the previous reports $[10,20]$. $\mathrm{KO}, \mathrm{HH}$, and WL had high percentage of VNS compared to other native chicken breeds, indicating that $\mathrm{KO}$ and $\mathrm{HH}$ have good semen quality.

Sperm morphology was recommended to be one of the most essential qualitative characteristics of poultry semen [23, 24]. The morphological defect rates observed in this study for KNCBs range from $8.8 \%$ to $16.0 \%$ that are in line with the earlier reports for different chicken breeds $[23,25,26]$. KO and WL had lower VAS and dead sperm rates, indicating that KO has the best semen quality for AI and semen cryopreservation. On the other hand, HY had the highest total morphological defect rate (16.0\%) followed by $\mathrm{HB}(11.7 \%)$. The highest proportions of the observed morphological defects were at the head and tail segments of the spermatozoa in all breeds, suggesting that these parts of spermatozoa are more susceptible to environmental factors. HY had significantly higher tail abnormality compared to $\mathrm{KO}$ and WL and even slightly higher compared to other native chicken breeds. The necessity for identifying specific abnormalities was emphasized in earlier study since some abnormalities may play a greater or lesser role in fertility [27]. The results of the present study show that bent, coiled, detached, broken, and knotted were common specific morphological defects in all breeds. The most frequent observed specific morphological defects on the head region of spermatozoa were: bent, broken, and knotted for $\mathrm{KO}$; detached, broken, and knotted for HB; detached, bent, and knotted for $\mathrm{HH}$; detached, bent, and coiled for HY; and bent, coiled, and knotted for WL. Bent, broken, and knotted were the most common observed midpiece abnormalities in all breeds. WL spermatozoa were observed to have more broken midpieces than native chicken breeds. The most common observed tail abnormalities were: bent and coiled for KO; broken and knotted for HB; bent and knotted for HH; bent and detached for HY; and coiled and Knotted for WL. The percentage of spermatozoa with coiled tail in $\mathrm{HH}$ was relatively low compared to other KNCBs. On the contrary, HY had the highest proportion of detached and bent but the lowest proportion of broken tail spermatozoa compared to other breeds.

In this study, the reported negative correlation of defective tail with MOT, fertility, and hatchability suggest that a high proportion of defective tail in an ejaculate volume will decrease MOT, fertility, and hatchability as these defects might affect the ability of the sperm to reach and penetrate an egg. However, the observed percent live abnormal sperm $(<17 \%)$ and dead sperm $(<10 \%)$ in fresh semen were too low to cause serious fertility and hatchability problems as evidenced by the reasonable fertility and hatchability results obtained in this study. Furthermore, almost all the observed morphological defects were secondary types of defects which were reported to be less serious compared to the primary type of defects and thought to arise during the passage through epididymis or by mishandling after ejaculation, suggesting the possibility of minimizing these defects through proper handling after ejaculation. Proper semen handling was reported to be essential to maintain optimum reproductive performance of AI programs [28]. Heritability estimates for fertility and hatchability in chickens were reported to be low [29], suggesting that the nongenetic factors have a higher influence on these traits. In this study, all breeds had reasonable fertility ( $87.8 \%$ to $96.4 \%)$ and hatchability (82.1\% to $92.9 \%)$ rates, however, $\mathrm{KO}$ and $\mathrm{WL}$ showed superior values for both traits compared to other $\mathrm{KNCBs}$. The highest $\mathrm{F}$ and $\mathrm{H}$ rates obtained for $\mathrm{KNCBs}$ in this study might partially be due to the use of WL hens for fertility and hatchability evaluation for all breeds. WL hens might have efficient/better oviductal sperm storage. Strain effects on oviductal sperm storage has been reported earlier $[8,30]$. The low motility and subsequent low fertility rates observed in HY may be associated with the combined effect of high rates of bent, coiled and detached defective head, and high rates of bent and detached tail defective of spermatozoa in an ejaculate volume of this breed.

\section{CONCLUSION}

This work provides valuable information on the association of live abnormal sperm and defective segments of spermatozoa with motility, fertility, and hatchability. Breed showed a significant effect on motility, dead sperm, VNS, VAS, and fertility and hatchability rates. Native chicken breeds' spermatozoa had more morphological defects than WL. Most observed morphological defects were at the head and tail in all breeds. VAS and defective tail had negative correlation with motility, fertility, and hatchability. HY spermatozoa were more susceptible to environmental factors. KO had best semen quality, fertility, and hatchability.

\section{CONFLICT OF INTEREST}

We certify that there is no conflict of interest with any financial organization regarding the material discussed in the manuscript. 


\section{ACKNOWLEDGMENTS}

This work was supported by National Research Foundation of Korea (NRF) grant (Project No. 2015R1A3A2033826) funded by the Ministry of Science, Information and Communication Technology, and Future Planning (MSIP), and the Cooperative Research Program for Agriculture Science and Technology Development (Project No. PJ012866012017) from the Korean Rural Development Administration.

\section{REFERENCES}

1. Sontakke SD, Umapathy G, Sivaram V, Kholkute SD, Shivaji S. Semen characteristics, cryopreservation, and successful artificial insemination in the Blue rock pigeon (Columba livia). Theriogenology 2004;62:139-53.

2. Alkan S, Baran A, Özadas ÖB, Evecev M. Morphological defects in turkey semen. Turk J Vet Anim Sci 2002;26:1087-92.

3. Sexton T. Breeding by artificial insemination. Br Poult Sci Ltd 1984:175-81.

4. Cheng FP, Guo TJ, Wu JT, et al. Annual variation in semen characteristics of pigeons (Columba livia). Poult Sci 2002;81: 1050-6.

5. Suh S, Sharma A, Lee S, et al. Genetic diversity and relationships of Korean chicken breeds based on 30 microsatellite markers. Asian-Australas J Anim Sci 2014;27:1399-405.

6. Malik A, Haron AW, Yusoff R, Nesa M, Bukar M, Kasim A. Evaluation of the ejaculate quality of the red jungle fowl, domestic chicken, and bantam chicken in Malaysia. Turk J Vet Anim Sci 2013;37:564-8.

7. Saacke R. Semen quality in relation to semen preservation. J Dairy Sci 1983;66:2635-44.

8. Stromberg J, Stromberg L. Guide to better hatching. Fort Dodge, IA, USA: Stromberg Pub. Co.; 1975.

9. Sexton T. Effect of semen treatments and age of tom on fertility of unstored semen and semen held 18 hours. Poult Sci 1987; 66:1721-6.

10. Haunshi S, Niranjan M, Shanmugam M, et al. Characterization of two Indian native chicken breeds for production, egg and semen quality, and welfare traits. Poult Sci 2011;90:314-20.

11. Shanmugam M, Rajkumar U, Reddy M, Rao SR. Effect of age on semen quality in naked neck and dwarf chicken under tropical climatic conditions. Anim Prod Sci 2012;52:964-8.

12. Scherf BD. World watch list for domestic animal diversity. Rome, Italy: Food and Agriculture Organization (FAO); 2000.

13. Burrows W, Quinn J. The collection of spermatozoa from the domestic fowl and turkey. Poult Sci 1937;16:19-24.

14. Khan M, Ijaz A. Effects of osmotic pressure on motility, plasma membrane integrity and viability in fresh and frozen-thawed buffalo spermatozoa. Animal 2008;2:548-53.

15. Björndahl L, Söderlund I, Kvist U. Evaluation of the one-step eosin-nigrosin staining technique for human sperm vitality assessment. Hum Reprod 2003;18:813-6.

16. Love C. Relationship between sperm motility, morphology and the fertility of stallions. Theriogenology 2011;76:547-57.

17. Pereira RJ, Napolitano A, Garcia-Pereira FL, et al. Conservation of avian germplasm by xenogeneic transplantation of spermatogonia from sexually mature donors. Stem cells Dev 2012;22:735-49.

18. Holt W. Basic aspects of frozen storage of semen. Anim Reprod SCci 2000;62:3-22.

19. Wishart G. Quantitation of the fertilising ability of fresh compared with frozen and thawed fowl spermatozoa. Br Poult Sci 1985;26:375-80.

20. Holsberger DR, Donoghue A, Froman D, Ottinger M. Assessment of ejaculate quality and sperm characteristics in turkeys: sperm mobility phenotype is independent of time. Poult Sci 1998;77:1711-7.

21. Nguyen TMD, Seigneurin F, Froment P, Combarnous Y, Blesbois E. The 5'-AMP-activated protein kinase (AMPK) is involved in the augmentation of antioxidant defenses in cryopreserved chicken sperm. PloS one 2015;10:e0134420.

22. Omeje S, Marire B. Evaluation of the semen characteristics of adult cocks of different genetic backgrounds. Theriogenology 1990;34:1111-8.

23. Lukaszewicz E, Jerysz A, Partyka A, Siudzinska A. Efficacy of evaluation of rooster sperm morphology using different staining methods. Res Vet Sci 2008;85;583-8.

24. Ntemka A, Tsousis G, Brozos C, et al. Breed differences of bull frozen-thawed semen. Reprod Domest Anim 2016;51: 945-52.

25. Kuster C, Singer R, Althouse G. Determining sample size for the morphological assessment of sperm. Theriogenology 2004; 61:691-703.

26. Hu J, Chen J, Wen J, et al. Estimation of the genetic parameters of semen quality in Beijing-You chickens. Poult Sci 2013;92: 2606-12.

27. Love CC, Varner DD, Thompson JA. Intra-and inter-stallion variation in sperm morphology and their relationship with fertility. J Reprod Fertil Suppl 1999:93-100.

28. Sharlip ID, Jarow JP, Belker AM, et al. Best practice policies for male infertility. Fertil Steril 2002;77:873-82.

29. Sapp R, Rekaya R, Misztal I, Wing T. Male and female fertility and hatchability in chickens: A longitudinal mixed model approach. Poult Sci 2004;83:1253-9.

30. Brillard J-P. Factors affecting oviductal sperm storage in domestic fowl following artificial insemination. Anim Reprod Sci 1992;27:247-56. 